\title{
Wireless Source Localization Utilizing An Airborne Interfrometry
}

\author{
Farzam Hejazi \\ Department of Electrical \\ and Computer Engineering \\ University of Central Florida \\ Orlando, USA \\ farzam.hejazi@ucf.edu
}

\author{
Mohsen Joneidi \\ Department of Electrical \\ and Computer Engineering \\ University of Central Florida \\ Orlando, USA \\ joneidi@knights.ucf.edu
}

\author{
Nazanin Rahnavard \\ Department of Electrical \\ and Computer Engineering \\ University of Central Florida \\ Orlando, USA \\ nazanin.rahnavard@ucf.edu
}

\begin{abstract}
This paper investigates the problem of localization of co-channel transmitters or primary users (PUs) using an array mounted on a moving aerial platform. As a practical alternative for a sensor network to pursue the localization task, the proposed Phase Interferometric Source Localization (PISL) technique utilizes a moving sensor that measures phase difference between two antennas mounted on the platform. Due to the sparse nature of PUs' distribution in the region, we model the localization task as a simple basis-pursuit denoising framework and introduce a reconstruction method using a sparse recovery algorithm to discover locations of unknown PUs based on the phase difference measurements. We show that the ratio of distance between two antennas to the carrier-frequency wavelength is the critical parameter making the localization feasible. We also propose a scheme for sensor motion design in order to maximize the number of detectable PUs based on mutual coherence property. Since the motion optimization problem is very hard to address we develop a simple geometric relaxation to address the problem. The simulation results show that PISL can precisely recover the map of PUs with only few measurements and also reveal that sensor motion path can have determinate effect on the localization accuracy. PISL performance is compared with an state-of-the-art technique that utilizes adaptive beamforming and results show the superiority of PISL results in localization accuracy.

Index Terms-Source Localization, Airborne platform, Sparse recovery, Mutual Coherence, Unmanned Aerial Vehicle (UAV), Cognitive radio networks and spectrum sensing.
\end{abstract}

\section{INTRODUCTION}

Localization of unknown and uncooperative PUs located in an area of interest has various practical civilian and military applications. In military utilization, Electronic Support (ES) systems require to know locations of enemies' PUs for electromagnetic environmental awareness and to prepare essential counter measures [1]-[4]. Recently, localization of primary users also has gained attention for spatial frequency reuse in cognitive radio applications. Bazerque et. al. introduced a technique for joint estimation of Power Spectral Density (PSD) in space and frequency in cognitive radio [5]. A cooperative network of sensors that measure signal strength is utilized to obtain measurements of signal's energy in arbitrary locations inside the network area. Consequently, using a basis expansion model of PSD in space and frequency, spatial estimation of PSD and PUs locations are acquired [6]. In this technique, it is supposed that channel gains magnitudes between sensors and
PUs are known. In another work, a more complex cooperative sensor network scheme was introduced that measures complex signals samples and utilizes a tensor-based method to address separation of power spectra and mapping the location of PUs [7]. In a recent work, sensors, each equipped with an array of antennas, steer direction of their beams adaptively for tracking already detected PUs and discovering new activities in area of interest [8].

In passive positioning literature, several methods proposed to pursue the localization task. A category of techniques uses direction-of-arrival (DOA) or angle-of-arrival (AOA) measurements to estimate target locations or to track a moving target [9]-[12]. In AOA-based positioning, a moving sensor or a sensor network should be employed [13], [14]. Time difference of arrival (TDOA) of received signals in a sensor network is widely used in literature to find locations of targets [15], [16]. Difference of doppler frequency received in different sensors also can be used to localize PUs in an environment [17], [18]. Lately, methods that use a combination of two or three of AOA/TDOA/FDOA techniques have been proposed [19], [20]. A category of techniques suggests to employ a moving platform to pursue passive source localization or direction finding. In [21], using a moving sensor, a large synthetic aperture antenna is formed to localize a simple harmonic source. In another work, a co-prime sampled large aperture array is employed to passively localize PUs [22]. A method similar to the Synthetic Aperture Radar (SAR) imaging technique introduced to passively detect and localize very low power PUs [23]. The proposed method suggest that the phase difference between received signals of two distant antennas mounted on a moving platform can be applied to find locations of unknown emitters in an area of interest [24], [25]. Using the same setup, we expand the results to multiple sources localization suggesting a simple receiver that uses a sparse recovery algorithm for the source localization task.

In state-of-the-art source localization techniques proposed in [5], [7] as the number of PUs increases in the area of interest, the number of sensors should be increased proportionally. Adding a group of connected sensors in a network is an exorbitant and time consuming mission and in most cases it is not feasible since it can't be done immediately. In our 
proposed method, instead of adding a bunch of sensors in arbitrary locations inside the network, we propose to employ a moving sensor to measure phase difference signals in divergent locations around the network. With the idea of using a moving sensor, not only we no longer need a sensor network but also we can reduce costs drastically since economical drones for surveillance missions are developing continuously [26], [27].

Our main contributions in this paper can be encapsulated as follows:

- introducing an airborne interferometric technique to measure phase difference signals in different points around an area of interest,

- modeling the localization task as a simple basis pursuit denoising problem using phase difference measurements

- investigating the condition of existence and uniqueness of the sparse solution and introducing an approximation of the localization resolution,

- developing a path optimization framework for the sensor to maximize the number of detectable PUs and suggesting a motion strategy for a simple square region of interest

The rest of the paper is ordered as follows. Section II formulate the localization problem as a linear equation system and propose an $\ell_{1}$ minimization sparse recovery solution to the problem. We analyze the conditions that make the localization feasible in section III. In section IV, we introduce a framework to optimize the sensor path in order to maximize the number of detectable PUs. Ultimately, Section V concludes the paper.

\section{Problem Formulation}

Finding PUs and their locations in a predefined region, referred as the area of interest is our main goal. Suppose there are $s \in \mathbb{N}$ primary active users positioned in the area of interest. We use a moving platform to pursue the source localization task. There are two omni-directional antennas mounted on the moving platform with distance $D$ that receive signals from PUs. The moving platform (sensor) measures the phase difference between its two antennas $m$ times during its movement. The 2D locations where the measurements are taken place are $l_{1}, l_{2}, \ldots, l_{m} \in \mathbb{R}^{2}$ and in time stamps $t_{1}, t_{2}, \ldots, t_{m} \in \mathbb{R}^{1}$, respectively. Figure 1 shows the geometry of the problem.

We define vector $\boldsymbol{b} \in \mathbb{C}^{m}$ to represent $m$ phase difference measurements

$$
\boldsymbol{b}=\left[\begin{array}{c}
\Delta\left(\phi\left(t_{1}\right)\right) \\
\Delta\left(\phi\left(t_{2}\right)\right) \\
\cdots \\
\Delta\left(\phi\left(t_{m}\right)\right)
\end{array}\right],
$$

where $\Delta\left(\phi_{i}\right), i \in\{1,2, \ldots, m\}$ is equal to the exponential phase difference signal between two antennas for the $i^{\text {th }}$ measurement. The phase difference signal is measured using the block diagram illustrated in Figure 2. To formulate the phase difference signal suppose that antennas 1 and 2 are receiving signals $z_{1}$ and $z_{2}$, respectively. Moreover, suppose that the $i^{\text {th }} \mathrm{PU}, i \in\{1,2, \ldots, S\}$ emits $S_{i}(t)$ given by

$$
S_{i}(t)=\bar{S}_{i}(t) \exp \left(-j 2 \pi f_{c} t\right)
$$

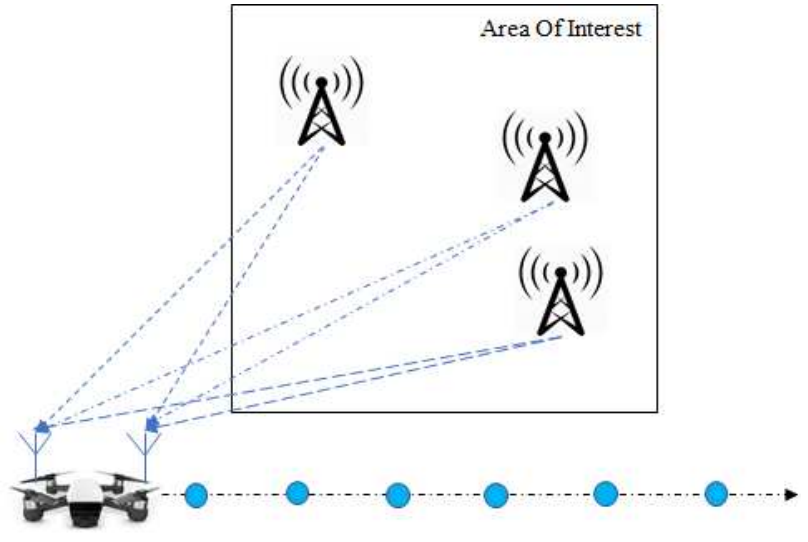

Fig. 1: Geometry of the problem. The blue circles show the sampling points. The moving platform takes $m$ samples along its way. Each sample is the phase difference between two received signals in its two antennas

where $f_{c}$ denotes the carrier frequency. Since we assume the PUs are co-channel, carrier frequencies are the same for all of the PUs. $\bar{S}_{i}(t)$ is the message or the waveform in the base band. Signals $z_{1}(t)$ and $z_{2}(t)$ are formulated as

$$
z_{1}(t)=\sum_{i=1}^{s} \alpha_{i}(t) S_{i}(t)+v_{1}(t),
$$

$$
z_{2}(t)=\sum_{i=1}^{s} \alpha_{i}(t) S_{i}\left(t+\Delta t_{i}(t)\right)+v_{2}(t),
$$

where $v_{1}(t)$ and $v_{2}(t)$ denote two independent random white Gaussian processes to represent noise and $\alpha_{i}$ models channel gain. $\Delta t_{i}(t)$ shows the time difference of arrivals in two antennas for the $i^{t h}$ signal. The cross-correlation between $z_{1}$ and $z_{2}$ can be calculated as

$$
\begin{aligned}
& \mathbb{E}\left\{z_{1}(t) z_{2}^{*}(t)\right\} \approx \\
& \sum_{i=1}^{s}\left|\alpha_{i}(t)\right|^{2} \mathbb{E}\left\{\bar{S}_{i}(t) \bar{S}_{i}^{*}\left(t+\Delta\left(t_{i}(t)\right)\right)\right\} \exp \left(-j 2 \pi f_{c} \Delta t_{i}(t)\right)+ \\
& \sum_{i=1}^{s} \sum_{k=1, i \neq k}^{s} \alpha_{i}(t) \alpha_{k}(t)^{*} \mathbb{E}\left\{\bar{S}_{i}(t) \bar{S}_{k}^{*}\left(t+\Delta t_{k}(t)\right)\right\} \exp \left(-j 2 \pi f_{c} \Delta t_{k}(t)\right) \\
& \quad+\mathbb{E}\left\{v_{1}(t) z_{2}^{*}(t)\right\}+\mathbb{E}\left\{v_{2}^{*}(t) z_{1}(t)\right\}+\mathbb{E}\left\{v_{2}^{*}(t) v_{1}(t)\right\} \text {. (4) }
\end{aligned}
$$

Assuming cross-correlations between $\bar{S}_{i}(t)$ s are zero [7], and massages $S_{i}(t)$ s are wide-sense stationary and ergodic, the phase difference signal is defined to be equal to the crosscorrelation between $z_{1}$ and $z_{2}$

$\Delta(\phi(t))=\mathbb{E}\left\{z_{1}(t) z_{2}^{*}(t)\right\}=\sum_{i=1}^{s} E_{i} \exp \left(-j 2 \pi f_{c} \Delta t_{i}(t)\right)$

where, 


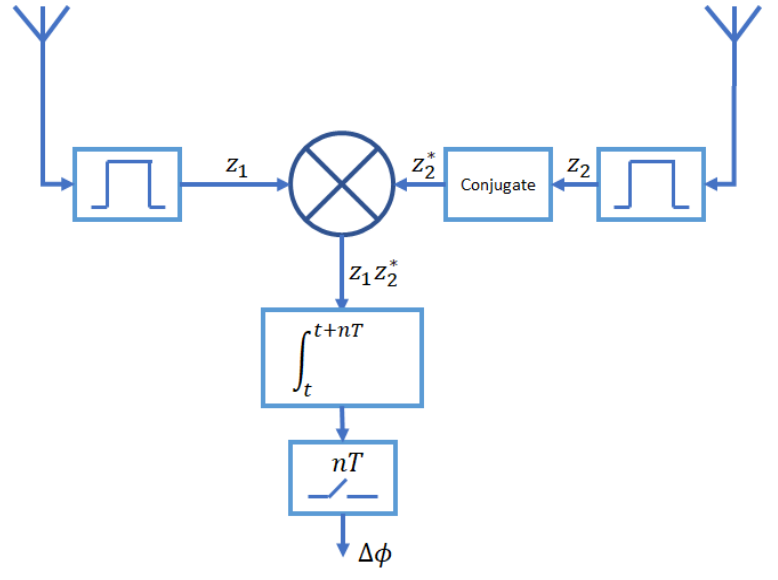

Fig. 2: Phase difference sampling task block diagram, since massages are assumed to be ergodic, the expectation task is approximated by a moving average in time domain

$$
E_{i}=\left|\alpha_{i}(t)\right|^{2} R_{\overline{S_{i}}}\left(\Delta\left(t_{i}(t)\right)\right),
$$

$R_{\bar{S}_{i}}(\tau)$ denotes the auto-correlation function of $\bar{S}_{i}$. Assuming $R_{\bar{S}_{i}}\left(\Delta\left(t_{i}(t)\right)\right) \approx R_{\bar{S}_{i}}(0)$, which means $\bar{S}_{i}$ is narrow-band, the remaining time-varying terms in (5) are exponential phase differences and channel gains $\left(\alpha_{i}(t)\right)$, these terms change very slowly in the time domain and can be considered constant in a short period of time (e.g. a second) [24]; therefore the expectation task in Figure 2 is not distorted by the timevarying nature of $\mathbb{E}\left\{z_{1}(t) z_{2}^{*}(t)\right\}$.

To find the potential PUs presented in a square area of interest we assume $n \times n$ identical and equally spaced cells cover the whole area, and there can be only a maximum of one PU located in each cell. We define vector $\boldsymbol{x} \in \mathbb{R}^{n^{2}}$ to indicate the presence of PUs in the region of interest. The $k^{t h}$ element of $\boldsymbol{x}$ is equal to $E_{k}{ }^{2}$ only if there is a PU in $k^{t h}$ cell otherwise it is zero. We define $\boldsymbol{A}=\left[a_{\ell k}\right] \in \mathbb{R}^{m \times n^{2}}$ to be a matrix that represents all possible phase difference measurements from all cells presented in the grid when phase difference measurements take place. Each column of $\boldsymbol{A}$ corresponds to a specific cell in the grid and represents $m$ expected phase difference measurements if there is a PU placed in the corresponding cell. Accordingly, the $a_{\ell k}(\ell \in\{1, \ldots, m\}$ and $\left.k \in\left\{1, \ldots, n^{2}\right\}\right)$ is given by

$$
a_{\ell k}=g_{\ell k} \exp \left(-j 2 \pi f_{c} \Delta t_{\ell k}\right),
$$

where $\Delta t_{\ell k}$ is the time difference of arrival of transmitted signal between two antennas in location $l_{\ell}$ if there is a PU in $k^{t h}$ cell of the grid. Also $g_{\ell k}$ is the channel gain between location $l_{\ell}$ and the $k^{\text {th }}$ cell. Here we suppose that the channel gain model is

$$
g_{\ell k}=\left|\alpha_{k}\left(t_{\ell}\right)\right|^{2}=\frac{\alpha}{r_{\ell k}^{3}},
$$

where $r_{k g}$ is the distance between $l_{k}$ and the $k^{t h}$ cell and $\alpha$ is a known attenuation parameter. Based on (5) the following linear equation system can be derived

$$
A x=b+\epsilon,
$$

where $\epsilon$ denotes the zero-mean error vector in phase difference measurements caused by irregularities in energy detector (Figure 2)

$$
\boldsymbol{\epsilon}=\left[\begin{array}{c}
\epsilon\left(t_{1}\right) \\
\epsilon\left(t_{2}\right) \\
\ldots \\
\epsilon\left(t_{m}\right)
\end{array}\right] .
$$

We add the noise term in (8), since the expectation step in (4) can't be performed ideally and noise terms in (4) can't be eliminated completely. The solution to the derived linear equation system in (8) is a typical basis pursuit solver. Given that $\boldsymbol{x}$ is sparse, it can be simply estimated by solving the following convex optimization problem

$$
\min _{\boldsymbol{x}}\|\boldsymbol{A} \boldsymbol{x}-\boldsymbol{b}\|_{2}^{2}+\lambda\|\boldsymbol{x}\|_{1}
$$

where $\lambda$ is a parameter that manage the trade-off between sparsity and reconstruction error. There are several solution proposed in the literature to solve the above convex optimization problem such as matching pursuit, in-crowd algorithm and homotopy continuation [28]-[30].

\section{A SuRVaY On Matrix $\boldsymbol{A}$}

Existence and uniqueness of a sparse solution to the linear system presented in (10) is directly related to properties of matrix $\boldsymbol{A}$. Restricted Isometry Property (RIP), Nullsapce and mutual coherence are among the most popular properties introduced in compressive sensing that define some conditions on $\boldsymbol{A}$ to guarantee a sparse solution [31]-[33]. Here we present a heuristic explanation of $\boldsymbol{A}$ 's mutual coherence property and conclude that the maximum correlation between columns is directly related to $\frac{D}{\lambda}$, where $\lambda$ denotes the wavelength at $f_{c}$. We define $\boldsymbol{a}_{i}, i \in\left\{1, \ldots, n^{2}\right\}$ to be the $i^{t h}$ column of $A$. Regarding the mutual coherence guarantee [33], if the maximum of $\left\langle\boldsymbol{a}_{i}, \boldsymbol{a}_{j}>, i \neq j\right.$ is close to zero, with a high probability (10) has a sparse solution. $\boldsymbol{a}_{i}$ represents $m$ expected phase difference measurements for the $i^{t h}$ cell in the $n \times n$ grid. We expect $\boldsymbol{a}_{j}$ which corresponds to the nearest cell, to have the maximum correlation with $\boldsymbol{a}_{i}$. Without loss of generality assume that $\boldsymbol{a}_{i}$ represents phase differences for the cell located at $\left(0, y_{i}\right)$. Also suppose that the moving sensor moves on $y=0$ line. Moreover presume $l_{i}=\left(\bar{x}_{i}, 0\right), i \in\{1, \ldots, m\}$. Refering (6) and using some simple approximations, $\boldsymbol{a}_{i}$ can be formulated as 
$\boldsymbol{a}_{i}=\left[\begin{array}{c}\frac{\alpha}{\left(y_{i}^{2}+\bar{x}_{1}^{2}+\frac{D^{2}}{4}\right)^{\frac{3}{2}}} \exp \left(-j \frac{2 \pi}{\lambda} \frac{D \bar{x}_{1}}{\sqrt{y_{i}^{2}+\bar{x}_{1}^{2}+\frac{D^{2}}{4}}}\right) \\ \frac{\alpha}{\left(y_{i}^{2}+\bar{x}_{2}^{2}+\frac{D^{2}}{4}\right)^{\frac{3}{2}}} \exp \left(-j \frac{2 \pi}{\lambda} \frac{D \bar{x}_{2}}{\sqrt{y_{i}^{2}+\bar{x}_{2}^{2}+\frac{D^{2}}{4}}}\right) \\ \ldots \\ \left(y_{i}^{2}+\bar{x}_{m}^{2}+\frac{D^{2}}{4}\right)^{\frac{3}{2}}\end{array}\right)$

Suppose that $\boldsymbol{a}_{\ell}$ corresponds to the cell located at $\left(\Delta x, y_{i}\right)$ right next to $i$ th cell in $x$-direction. Therefore the correlation between $\boldsymbol{a}_{i}$ and $\boldsymbol{a}_{\ell}$ is equal to

$<\boldsymbol{a}_{i}, \boldsymbol{a}_{\ell}>\approx \frac{\alpha\left|\sum_{k=1}^{m} \frac{1}{\left(y_{i}^{2}+{\overline{x_{k}}}^{2}\right)^{3}} \exp \left(j 2 \pi \frac{D}{\lambda} \frac{\Delta x}{\sqrt{y_{i}^{2}+\overline{x k}^{2}}}\right)\right|}{\left(\sum_{k=1}^{m} \frac{1}{\left(y_{i}^{2}+\bar{x}^{2}\right)^{\frac{3}{2}}}\right)^{2}}$

in which $\sqrt{y_{i}^{2}+{\overline{x_{k}}}^{2}}$ is equal to the distance between the $i^{\text {th }}$ cell and the sensor in the $k^{t h}$ measurement. Numerator (12) is a summation of some weighted exponential complex phases that change between $\left(2 \pi \frac{D}{\lambda} \frac{\Delta x}{r_{\max }}, 2 \pi \frac{D}{\lambda} \frac{\Delta x}{r_{\min }}\right)$, in which $r_{\min }$ and $r_{\max }$ are the minimum and maximum distances between the moving sensor and the $i^{\text {th }}$ cell. To simplify the analysis, if $r_{\max }$ is large enough and $r_{\max } \gg r_{\min }$ then $2 \pi \frac{D}{\lambda} \frac{\Delta x}{r_{\max }} \approx 0$.

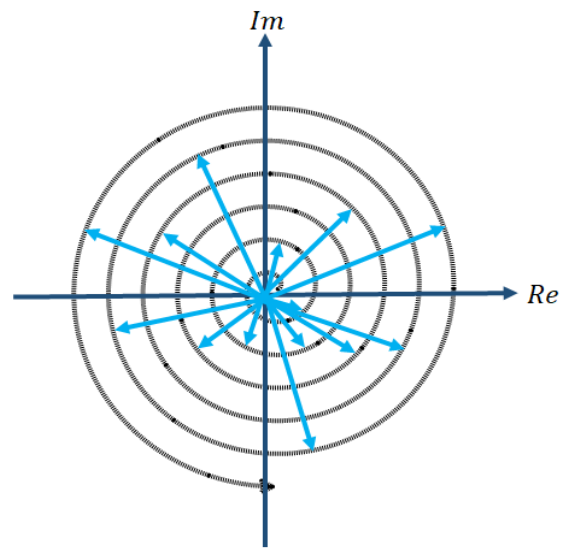

Fig. 3: Summation of vector with arbitrary amplitudes, will be more close to zero if their phases change between $(0,2 k \pi)$ rather than vectors that their phases are close to each other
It can be claimed that if the phase changes between $(0,2 \pi)$ or $(0,2 k \pi), k \in \mathbb{N}$ and assuming that phases selected arbitrarily in this interval, then it is expected that the summation of complex exponential phases in (12) is in the vicinity of zero. In other words, if $\Delta x=k \frac{\lambda r_{\text {min }}}{D}, k \in \mathbb{N}$ then it is expected that the summation is close to zero (Figure 3). A similar argument holds for the nearest cell to the $i^{t h}$ cell in $\mathrm{y}$-direction and it can be reasoned that if $\Delta y \propto k \frac{\lambda r_{\text {min }}}{D}$ then $\boldsymbol{a}_{i}$ and $\boldsymbol{a}_{j}$ are almost uncorrelated. These results are consistent with the image resolution derivation in azimuth and range directions derived in [24], in which it is proved the resolution of the proposed imaging method in both azimuth and range directions are in proportion to $\frac{\lambda r_{\min }}{D}$ if the sensor moves along a straight line. To sum up, we argue that if the moving platform proceeds in a straight line and samples the phase difference regularly, then the feasibility of the recovery of PUs locations depends on $\frac{\lambda r_{\min }}{D}$. In the following section, we expand a similar discussion to reach a framework for sensor path optimization.

\section{A Framework fOR PATH Optimization}

Previous section reveals that sampling locations have a determinate effect on the sparse solution of (8). In this section, we introduce a scheme that can be applied for the selection of sampling points and consequently motion design of the moving sensor. With reference to [33], [34], if the number of targets in the area of interest is at most $\frac{1}{2}\left(1+\mu^{-1}(A)\right)$, where

$$
\mu(A)=\max _{i, j \in\{1, \ldots, m\}, i \neq j} \frac{\left|\left\langle\boldsymbol{a}_{i}, \boldsymbol{a}_{j}\right\rangle\right|}{\left\|\boldsymbol{a}_{i}\right\|_{2} \cdot\left\|\boldsymbol{a}_{j}\right\|_{2}},
$$

then the $l_{1}$ minimization can reach a sparse solution to discover them. To maximize number of detectable PUs the $\mu(A)$ should be minimized. As a result, the path optimization problem can be turns into minimization of maximum mutual coherence between columns of $\boldsymbol{A}$

$$
\min \max _{i, j \in\{1, \ldots, m\}, i \neq j} \frac{\left|\left\langle\boldsymbol{a}_{i}, \boldsymbol{a}_{j}\right\rangle\right|}{\left\|\boldsymbol{a}_{i}\right\|_{2} \cdot\left\|\boldsymbol{a}_{j}\right\|_{2}},
$$

We enumerate cells as $i=i_{\text {row }} n+i_{\text {col }} ; 0<i_{\text {row }}<n-1$ and $0<i_{\text {col }}<n-1$, denote row and column numbers of cell $i$, respectively. Also suppose that the first cell is located in $(0,0)$ and assume that all cells are placed equally spaced in both $x, y$ directions. So the $i^{\text {th }}$ cell is located at $\left(i_{\text {col }} \Delta x, i_{\text {row }} \Delta y\right)$ where $\Delta x$ and $\Delta y$ is equal to the minimum distance between cells in $x$ and $y$ direction respectively. Phase differences samples taken at $l_{i}=\left(x_{i}, y_{i}\right), i \in\{1, \ldots, m\}$ locations. Moreover, the first and the second antennas are located at $l_{a_{1 i}}=\left(x_{i}-\right.$ $\left.\frac{D_{x_{i}}}{2}, y_{i}-\frac{D_{y_{i}}}{2}\right)$ and $l_{a_{2 i}}=\left(x_{i}+\frac{D_{x_{i}}}{2}, y_{i}+\frac{D_{y_{i}}}{2}\right)$, where $D=$ $\sqrt{D_{x_{i}}^{2}+D_{y_{i}}^{2}}, i \in\{1, \ldots, m\}$, respectively. We represent $r_{k i}$ and $r_{k j}$ to be the vector the connects the sensor and centers of $i^{t h}$ and $j^{t h}$ cells in the $k^{t h}$ measurement

$$
r_{k i}=\left\|r_{k i}\right\|_{2}=\sqrt{\left(x_{k}-i_{c o l} \Delta x\right)^{2}+\left(y_{k}-i_{\text {row }} \Delta y\right)^{2}},
$$




$$
r_{k j}=\left\|r_{k j}\right\|_{2}=\sqrt{\left(x_{k}-j_{c o l} \Delta x\right)^{2}+\left(y_{k}-j_{\text {row }} \Delta y\right)^{2}} .
$$
form

Hence, the vector $\boldsymbol{a}_{i}$ can be formulated in the following

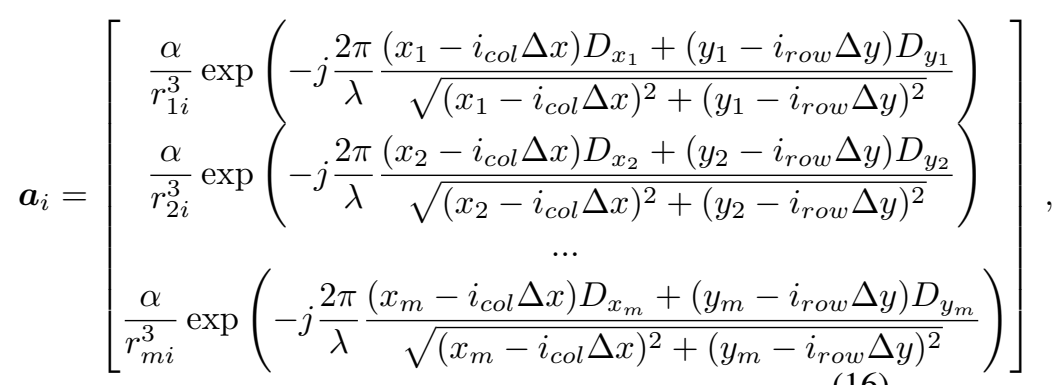

also we define $\chi$

$$
\boldsymbol{\chi}=\left[\begin{array}{c}
\left(x_{1}, y_{1}, D_{x_{1}}, D_{y_{1}}\right) \\
\left(x_{2}, y_{2}, D_{x_{2}}, D_{y_{2}}\right) \\
\ldots \\
\left(x_{m}, y_{m}, D_{x_{m}}, D_{y_{m}}\right)
\end{array}\right]
$$

to represent locations of two antennas in all measurements' points. Accordingly, the optimization problem in (14) turns into the following optimization problem

$$
\begin{aligned}
& \min _{\chi} \max _{i, \ell \in\left\{1, \ldots, n^{2}\right\}, i \neq \ell} \frac{1}{\sqrt{\sum_{k=1}^{m} \frac{1}{R_{k i}^{6}}} \sqrt{\sum_{k=1}^{m} \frac{1}{R_{k \ell}^{6}}}} \\
& \quad \mid \sum_{k=1}^{m} \frac{1}{R_{k i}^{3} R_{k \ell}^{3}} \exp \frac{-j 2 \pi}{\lambda}\left\{\frac{\left(x_{k}-i_{\text {col }} \Delta x\right) D_{x_{k}}+\left(y_{k}-i_{\text {row }} \Delta\right.}{\sqrt{\left(x_{k}-i_{\text {col }} \Delta x\right)^{2}+\left(y_{k}-i_{\text {row }} \Delta\right.}}\right) \\
& \left.\quad-\frac{\left(x_{k}-\ell_{\text {col }} \Delta x\right) D_{x_{k}}+\left(y_{k}-\ell_{\text {row }} \Delta y\right) D_{y_{k}}}{\sqrt{\left(x_{k}-\ell_{\text {col }} \Delta x\right)^{2}+\left(y_{k}-\ell_{\text {row }} \Delta y\right)^{2}}}\right\} \mid \\
& \text { subject to: } D_{x_{i}}^{2}+D_{y_{i}}^{2}=D^{2}
\end{aligned}
$$

The above optimization problem is very hard to analyze. We try to manipulate a geometric explanation that helps us to suggest a solution to the optimization problem.

To find the justification let us formulate $\boldsymbol{a}_{i}$ again. The inner term in each element of $\boldsymbol{a}_{i}$ in (16) can be simplified to inner product of two vectors

$$
\begin{array}{r}
\frac{\left(x_{k}-i_{c o l} \Delta x\right) D_{x_{k}}+\left(y_{k}-i_{\text {col }} \Delta y\right) D_{y_{k}}}{\sqrt{\left(x_{k}-i_{c o l} \Delta x\right)^{2}+\left(y_{k}-i_{\text {row }} \Delta y\right)^{2}}} \\
=\frac{\left(D_{x_{k}}, D_{y_{k}}\right)^{T}\left(x_{k}-i_{c o l} \Delta x, y_{k}-i_{\text {row }} \Delta y\right)}{\left\|\left(x_{k}-i_{\text {col }} \Delta x, y_{k}-i_{\text {row }} \Delta y\right)\right\|_{2}} \\
=D \cos \left(\theta_{k i}\right),
\end{array}
$$

where $\theta_{k i}$ is the angle between vector $\overrightarrow{d_{k}}=\left(D_{x_{i}}, D_{y_{i}}\right)$ and vector $r_{k i}$. As Figure 4 illustrates vector $\overrightarrow{d_{k}}$ is a vector that connects the first and the second antenna in the $k^{\text {th }}$ measurement. Consequently, we can reformulated the minmax problem of

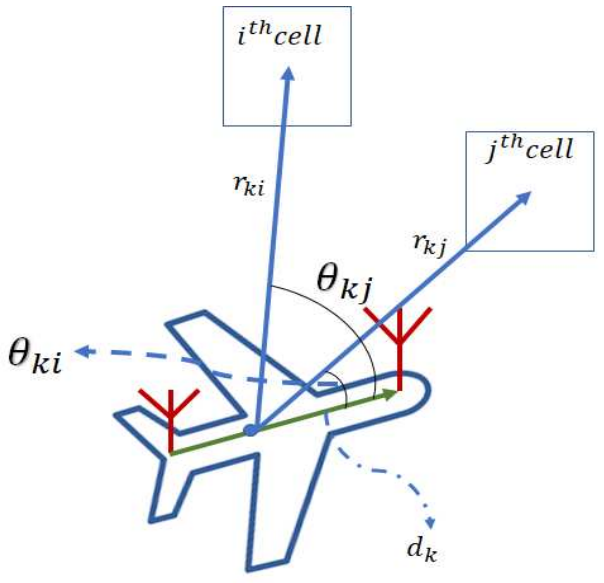

Fig. 4: Illustration of $\vec{r}_{k i}, \vec{r}_{k j}, \vec{d}_{k}, \theta_{k i}, \theta_{k j}$
(18) as the following form

$$
\begin{gathered}
\min _{\chi} \max _{i, \ell \in\left\{1, . ., n^{2}\right\}, i \neq j} \frac{1}{\sqrt{\sum_{k=1}^{m} \frac{1}{r_{k i}^{6}}} \sqrt{\sum_{k=1}^{m} \frac{1}{r_{k \ell}^{6}}}} \\
\left|\sum_{k=1}^{m} \frac{1}{r_{k i}^{3} r_{k \ell}^{3}} \exp \left(-j \frac{2 \pi D}{\lambda}\left(\cos \theta_{k i}-\cos \theta_{k \ell}\right)\right)\right|,
\end{gathered}
$$

The summation in (20) is a summation of weighted exponential phases that changes between

$\frac{2 \pi D}{\lambda}\left(\min _{k \in\{1, \ldots, m\}}\left|\cos \theta_{k i}-\cos \theta_{k \ell}\right|, \max _{k \in\{1, \ldots, m\}}\left|\cos \theta_{k i}-\cos \theta_{k \ell}\right|\right)$,

$\Delta^{\mathrm{F}}{ }^{2}$ the minimum part in (21), if $\theta_{k i}=\theta_{k \ell}$ then the minimum is zero and for the maximum part, we demonstrate the following Theorem.

Theorem 1. If phase difference samples being measured along a straight line parallel to the line that connects centers of two cells $(i, j)$, then

$$
\max _{k \in\{1, \ldots, m\}}\left|\cos \theta_{k i}-\cos \theta_{k j}\right| \leq \frac{\Delta x}{\sqrt{r_{\text {min }}^{2}+\frac{\Delta_{x}^{2}}{4}}},
$$

where $\Delta x$ is the distance between the center of the two cells and $r_{\min }$ is the distance between the line that connects centers of two cells and the sensor movement line.

Proof. Regarding (19) $\left|\cos \theta_{k i}-\cos \theta_{k j}\right|$ can be written as

$$
\begin{array}{r}
\left|\cos \theta_{k i}-\cos \theta_{k j}\right|=\frac{1}{D}\left|\frac{\overrightarrow{d_{k}} \cdot r_{k i}}{\left\|r_{k i}\right\|_{2}}-\frac{\overrightarrow{d_{k}} \cdot r_{k j}}{\left\|r_{k j}\right\|_{2}}\right|_{2} \\
=\frac{1}{D}\left|\overrightarrow{d_{k}} \cdot\left(\frac{r_{k i}}{\left\|r_{k i}\right\|_{2}}-\frac{r_{k j}}{\left\|r_{k j}\right\|_{2}}\right)\right|_{2} \\
\leq \frac{\left|\overrightarrow{d_{k}}\right|_{2}}{D}\left|\left(\frac{r_{k i}}{\left\|r_{k i}\right\|_{2}}-\frac{r_{k j}}{\left\|r_{k j}\right\|_{2}}\right)\right|_{2} \\
=\left|\frac{r_{k i}}{\left\|r_{k i}\right\|_{2}}-\frac{r_{k j}}{\left\|r_{k j}\right\|_{2}}\right|_{2},
\end{array}
$$


where $\frac{r_{k} i}{\left\|r_{k i}\right\|_{2}}$ and $\frac{r \vec{k} j}{\left\|r_{k j}\right\|_{2}}$ are 2 unit vectors and the angle between these two vector is $\angle\left(\theta_{k i}-\theta_{k j}\right)$. $\left|\frac{r_{k} i}{\left\|r_{k i}\right\|_{2}}-\frac{r_{\vec{k} j}}{\left\|r_{k j}\right\|_{2}}\right|_{2}$ is maximized when the angle between the two vector maximized.

Lemma 1. Suppose that line $\mathrm{x}$ in parallel to segment $\overline{A B}$ (Figure 5), and suppose that $C$ is an arbitrary point on the line $\mathrm{x}$. The angle $\angle A C B$ is maximized when $\overline{A C}=\overline{B C}$.

Proof. Figure 5 shows the geometric configuration of the problem, suppose that point $D$ placed on the line $x$ such that $\overline{A D}=\overline{B D}$ and $C$ is an arbitrary point on the line $x$. To porve Lemma 1, we prove that $\angle \beta \geq \angle \alpha$. Consider triangle $\triangle C B D$ and $\triangle A C D$, applying the law of sines we have

$$
\begin{gathered}
\triangle C B D: \frac{\overline{C D}}{\sin \eta}=\frac{\overline{B D}}{\sin \gamma}=\frac{\overline{B C}}{\sin (\kappa+\beta)}, \\
\triangle C A D: \frac{\overline{C D}}{\sin \zeta}=\frac{\overline{A D}}{\sin (\gamma+\alpha)}=\frac{\overline{B D}}{\sin (\gamma+\alpha)}=\frac{\overline{A C}}{\sin \kappa},
\end{gathered}
$$

regarding $\kappa=\frac{\pi}{2}-\frac{\beta}{2}$, then it is easy to show that $\sin (\kappa+\beta)=$

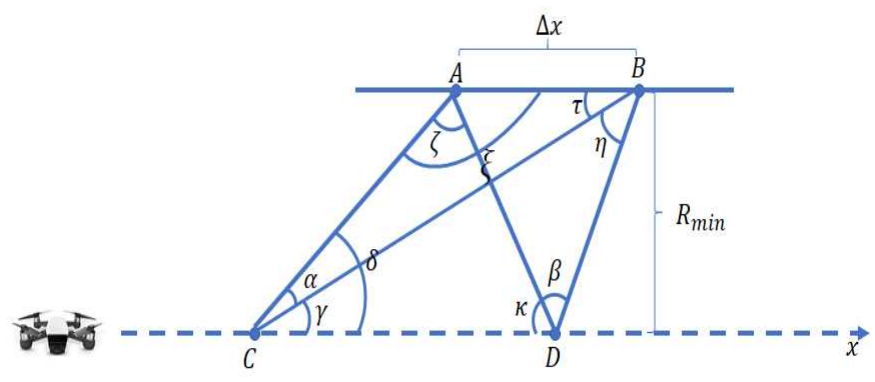

Fig. 5: Geometry of the problem in Lemma 1, $A, B$ are centers of two cells $(i, j)$, line $x$ is the platform path, $\overline{A D}=\overline{B D}$, and $C$ is an arbitrary point on line $x$

$\sin (\kappa)$. We can formulated $\sin (\eta)$ and $\sin (\zeta)$ as follows

$$
\begin{gathered}
\sin \eta=\frac{\overline{C D}}{\overline{B C}} \sin \kappa \\
\sin \zeta=\frac{\overline{C D}}{\overline{A C}} \sin \kappa,
\end{gathered}
$$

in triangle $\triangle A C B$

$$
\angle \xi>\angle \tau+\angle \eta>\angle \tau \rightarrow \overline{B C}>\overline{A C},
$$

(25) and (26) result in $\sin \zeta>\sin \eta$ and if $\zeta<\frac{\pi}{2}$ we can conclude that $\zeta>\eta$. If $\zeta>\frac{\pi}{2}$ since $\eta<\frac{\pi}{2}$ again we have $\zeta>\eta$. Regarding $\angle \alpha+\zeta=\angle \eta+\beta$, finally we can conclude that $\angle \beta>\angle \alpha$.

Using Lemma $1, \frac{r_{\vec{k} i}}{\left\|r_{k i}\right\|_{2}}-\frac{r_{\vec{k} j}}{\left\|r_{k j}\right\|_{2}}$ is maximized when $\left\|r_{k j}\right\|_{2}=\left\|r_{k i}\right\|_{2}$. Respecting to (23), $\left|\cos \theta_{k i}-\cos \theta_{k j}\right|$ is maximized when $\vec{D}$ and $\frac{r_{\vec{k} i}}{\left\|r_{k i}\right\|_{2}}-\frac{r_{\vec{k} j}}{\left\|r_{k j}\right\|_{2}}$ are in the same direction and also $\left\|r_{k j}\right\|_{2}=\left\|r_{k i}\right\|_{2}$. Both of these conditions satisfy when the moving platform passes the point $D$ in Figure 5 , therefore

$$
\begin{array}{r}
\left|\cos \theta_{k i}-\cos \theta_{k j}\right|=\left|\frac{r_{k i}}{\left\|r_{k i}\right\|_{2}}-\frac{r_{k j}}{\left\|r_{k j}\right\|_{2}}\right|_{2} \\
=\frac{1}{\left\|r_{k i}\right\|_{2}}\left\|r_{k i}-r_{k j}\right\|=\frac{\Delta x}{\left\|r_{k i}\right\|_{2}}=\frac{\Delta x}{\sqrt{r_{\min }^{2}+\frac{\Delta x^{2}}{4}}},
\end{array}
$$

so we can conclude that,

$$
\max _{k \in\{1, \ldots, m\}}\left|\cos \theta_{k i}-\cos \theta_{k j}\right| \leq \frac{\Delta x}{\sqrt{r_{\text {min }}^{2}+\frac{\Delta_{x}^{2}}{4}}},
$$

Corollary 1. For any two arbitrary cells $(i, j)$ inside the grid, the following inequality holds

$$
\max _{k \in\{1, \ldots, m\}}\left|\cos \theta_{k i}-\cos \theta_{k j}\right| \leq \frac{\Delta x}{r_{\min }},
$$

where $\Delta x$ is the distance between the center of two cells.

Proof. Regarding Theorem 1. for each sampling point the above inequality holds and consequently, if $r_{\min }$ is the global minimum distance between the platform and the center line of two cells, subsequently the above Corollary holds.

With respect to Corollary 1 , we expect that two cells that placed next to each other have the minimum changes in phase interval (21). Now suppose that the $i^{t h}$ and $j^{t h}$ cells are located next to each other. If the platform is far enough from these two cells (Figure 6(c)) or sample at the moment when the sensor located in the line that connects the centers of the two cells (Figure 6(b)), then the $\min _{k \in\{1, \ldots, m\}}\left|\cos \theta_{k i}-\cos \theta_{k j}\right|=0$. The maximum occurs when the following conditions happens(Figure 6(a)):

- sensor samples the phase difference when located at the perpendicular bisector of the center of two cells

- $\vec{D}$ is perpendicular to the perpendicular bisector line

- The distance between the platform and the two cells is minimized in the sampling moment and equal to the $r_{\min }$ Consequently, the maximum in (21) turns into

$$
\max _{k \in\{1, \ldots, m\}}\left|\cos \theta_{k i}-\cos \theta_{k j}\right| \approx \frac{\Delta x}{r_{\min }},
$$

where $\Delta x$ is the distance between the two cell. Therefore, the interval in (21) is a subset of $\left(0,2 \pi \frac{D}{\lambda} \frac{\Delta x}{r_{\text {min }}}\right)$.

It can be claimed that if $\frac{D}{\lambda} \frac{\Delta x}{r_{\min }}=k, k \in \mathbb{N}$ and if minimum and maximum of $\left|\cos \theta_{k i}-\cos \theta_{k j}\right|$ is equal or close to $\left(0, \frac{\Delta x}{r_{\text {min }}}\right)$ and if the sensor measures some arbitrary phases in the interval then it is very probable that the summation in (20) is close to zero. Regarding the boundary $\frac{1}{2}\left(1+\mu^{-1}(A)\right)$ for number of detectable targets, with a high probability any sparse distribution of PUs in the area of interest that meets or close to the bound can be recovered with PISL. For a special practical case, assume that the area of interest is a square region and cells that shape the grid are square and equally spaced in the region. Then a good strategy for sampling that satisfies the upper and lower bound for $\left|\cos \theta_{k i}-\cos \theta_{k j}\right|$ is that the sensor moves along all four edge of the region 
(a)

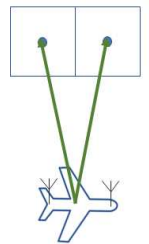

(c)

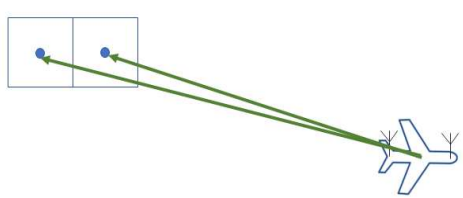

Fig. 6: (a) The scenario that maximize $\left|\cos \theta_{k i}-\cos \theta_{k j}\right|$, (b),(c) scenarios that minimize $\left|\cos \theta_{k i}-\cos \theta_{k j}\right|$

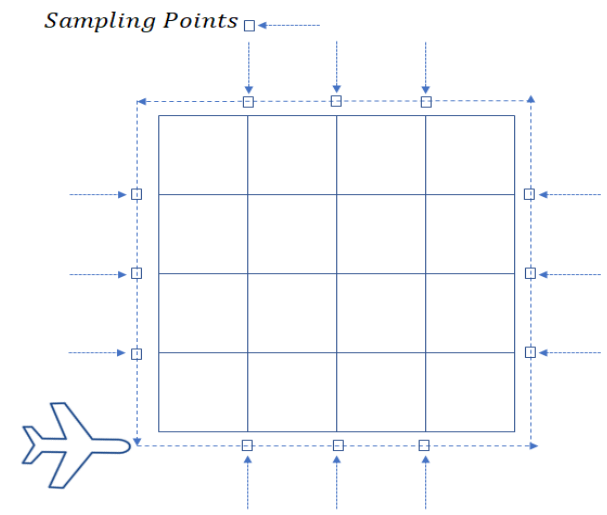

Fig. 7: Recommended path and sampling points for a square region

and samples the phase difference on the borders that separate cells along each edge (Figure (7)). The recommended strategy guarantees the minimum and maximum of $\left|\cos \theta_{k i}-\cos \theta_{k j}\right|$ to be 0 and $\frac{\Delta x}{r_{\min }}$ for each two cells that placed next to each other along each edge. Also we have enough measurements between 0 and $\frac{\Delta x}{r_{\min }}$ that puts the summation in (20) in vicinity of zero.

\section{Simulations}

Here we examine the proposed idea with numerous simulations and try to test PISL from different points of view and compare it with the method proposed in [8]. In the first simulation, we suppose that there are several PUs presented in the area of interest and there is a moving sensor that senses the area with two omni-directional antennas mounted on it with $D=5$ meters distance between them. We suppose that the carrier frequency of the primary users are $f_{c}=10 \mathrm{GHz}$. The area of interest is a square $10 \mathrm{Km} \times 10 \mathrm{Km}$ area and to find locations of potential PUs we hypothetically cover the area with a $30 \times 30$ grid. The moving platform moves around the area of interest only once and gathers $m$ samples of phase difference between two antennas in equally spaced steps. In the first simulation, we examine whether the proposed receiver is capable of recovering PUs'(or PUs) locations. The number of PUs in the region is 30 (show by $\diamond$ in Figure 8 ) and the sensor takes $m=100$ measurements and the signal-to-noise-ratio (SNR) is $22 \mathrm{~dB}$. Figure 8 illustrates that the proposed sparse recovery method can recover locations of PUs up-to the grid that they belong in. Interestingly, we recover a location map with 900 grid points, based on only 100 measurements due to the sparse nature of emitters' locations. As the figure illustrates PISL can even distinguish between targets that located in cells right next to each other.

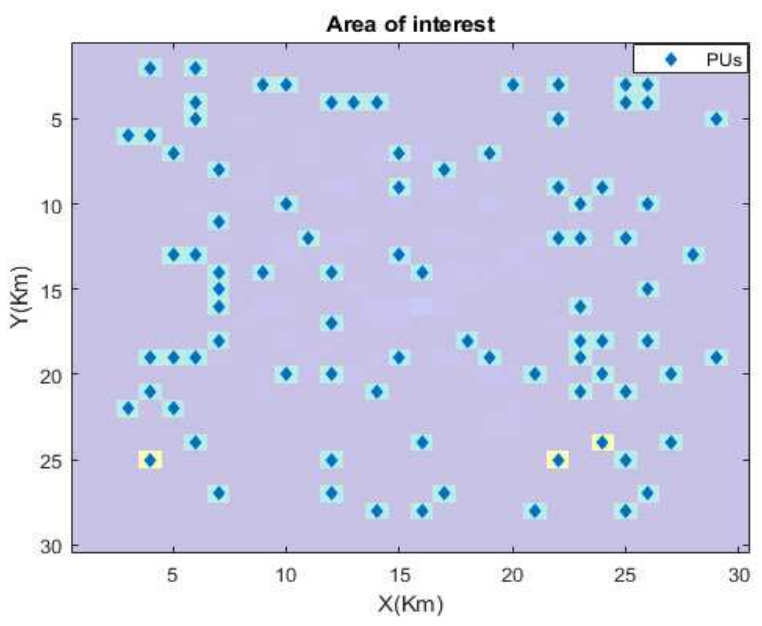

Fig. 8: An area of interest contours 30 transmitters (PUs whose location is in blue $\diamond$ ). The area is assumed to be tiled into 900 grid points, yellow and green grids show the estimated location of PUs with different transmitting powers

In the next simulation the number of Primary users is 6 and number of measurements is 100. Figure 9 shows the recovered location of PUs along with the recovered power map. Concerning equation (5) and equation (7) the power of PUs can be estimated and the corresponding power map can be pictured.

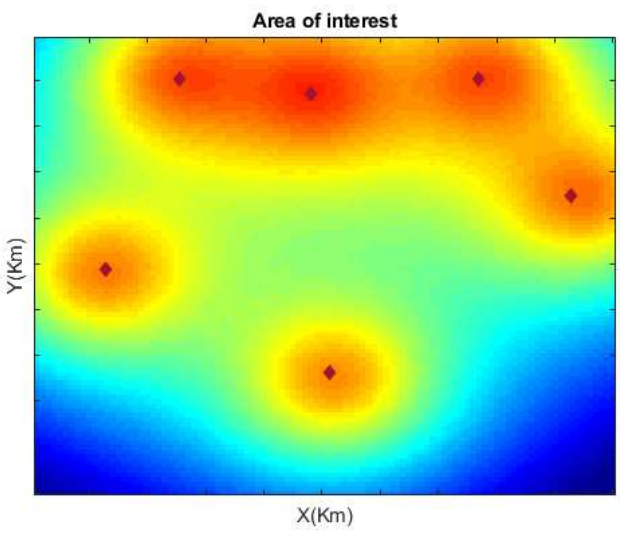

Fig. 9: Recovered locations of PUs (in red $\diamond$ ) and the recovered power map when there are more sources presented. 
One of the main concerns in applying the proposed recovery algorithm is noise. We define $e$ to represent the normalized estimation error

$$
e=\frac{\|\boldsymbol{x}-\overline{\boldsymbol{x}}\|_{2}}{\|\boldsymbol{x}\|_{2}},
$$

where $\overline{\boldsymbol{x}}$ is the recovered sparse vector in (10). In the following simulation we examine the effect of the input SNR on the estimation error. We assume that there is only 2 PUs presented in the area of interest and the number of measurements is 20 and a $10 \times 10$ grid.

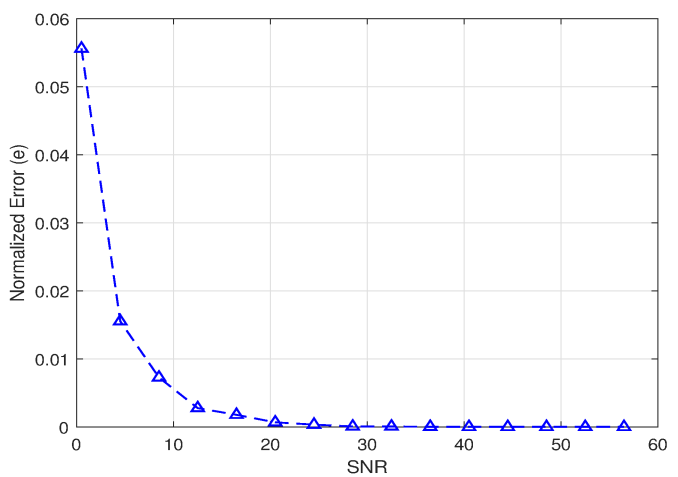

Fig. 10: Normalized estimation error versus input SNR (dB). In this scenario, $\mathrm{n}=10$ and $\mathrm{s}=2 \mathrm{~m}=20$.

Figure 10 shows that the estimation error is a decreasing function of input SNR, as expected. Regarding the figure, the normalized error oversteps 0.01 for SNRs less than $6 d B$. Regarding (4), since there is an integration process before sampling in many practical cases higher SNRs is achievable. Another parameter which is important is the number of measurement needed to recover locations of PU in the area of interest. In the following simulation we plot estimation error versus the number of measurements $m$. We assume that number of sources are 8 and the input SNR equal to $20 \mathrm{~dB}$.

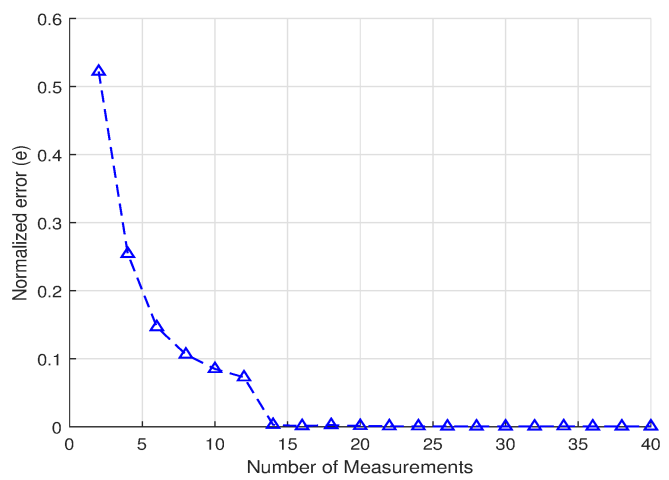

Fig. 11: Error in estimating vector $x$ versus input number of measurements. In this scenario, $n=10$ and $s=8$

Figure 11 demonstrates that as the number of measurements increases, output error drops and after 14 measurements the output error converge to 0.001 . In the next simulation, we will analyze the effect of $\frac{D}{\lambda}$ on the estimation error.

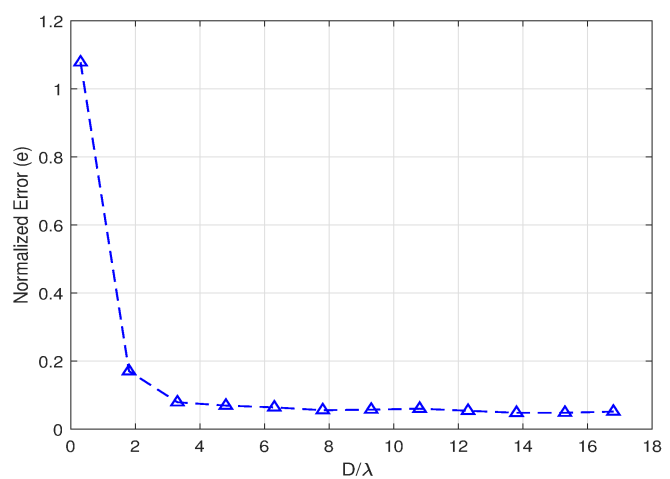

Fig. 12: Effect of $D / \lambda$ on the estimation error of vector $x$, $m=25, s=8, n=10$

In this simulation we assume that sensor moves along one edge of the region. Figure 12 illustrates that the estimation error drops to its minimum possible for $D / \lambda$ greater than 3 . This is compatible with the results presented in Sections III and IV that the proportion $\frac{D}{\lambda}$ has a determining effect on localization accuracy. It should be mentioned that in many radio interferometric imaging applications it has been shown that the $\frac{D}{\lambda}$ plays a key role in determining the imaging system resolution, where $D$ is the antenna aperture [35], [36].

In the following simulation, we consider two types of motion, the first one is in the direction of one edge of the region and the second one is a square around the region of interest (Figure 7). The number of measurements are equal in the both motion strategies and all sampled in equally-spaced steps. Figure 13 clarify that by the motion strategy in which the platform moves along an edge of a region of interest the estimation error will about 10 times more than the error of the strategy that select to move around the region (section IV), after 15 measurements has been taken.

In the final simulation, we compare PISL performance with the state-of-the-art technique presented in [8]. The method utilizes a sensor network, each sensor is equipped with an array antenna -instead of set of omni-directional energy detector suggested in [5] and step by step arrays' beams are adapted to increase localization accuracy. Due to utilizing adaptive beamforming, the method reaches much lower estimation error compare to the method presented in [5]. In the simulation, we assume the moving sensor moves in a square around the area of interest, and we also presume that fixed sensors are exactly located at the moving sensor sampling points which means the network has $m$ sensors, each equipped with an $M_{a}$-array antenna. Also we set $m=10, s=5, S N R=26 d B$.

In Figure 14, at each time steps the sensor network adapts beams' patterns to reach better locations' estimations. The Figure elucidates that with $M=16$ the error of two techniques are comparable, however, PISL reached more precise estimates. Although only two antennas are implemented in 


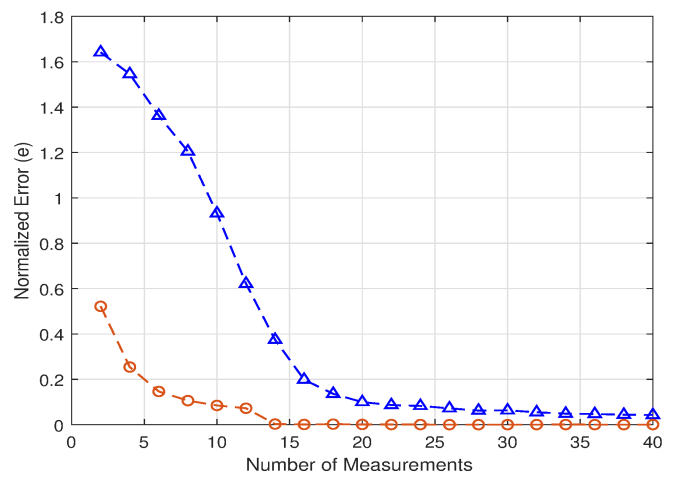

Fig. 13: Comparison between 2 type of sensor motion in estimation error, first strategy is moving along an edge of the area of interest (marked with triangle) and the second strategy is moving around it (marked with circle), $s=8, n=10$, $S N R=20 d B$

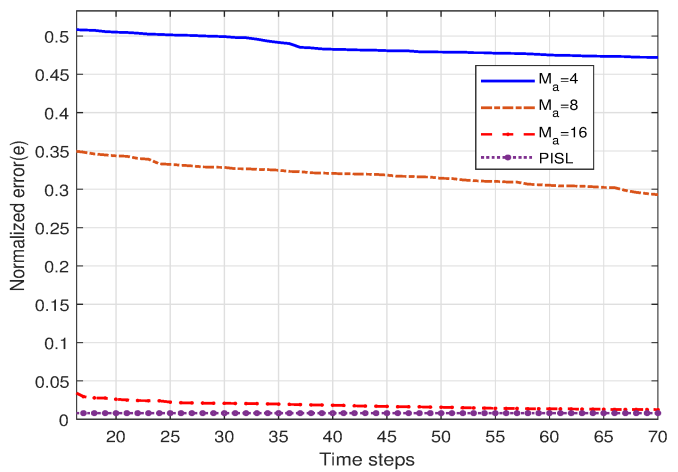

Fig. 14: Estimation error when the there is a sensor network with $M_{a}$-array antennas in comparison with a moving sensor $M_{a}=\{4,8,16\}, m=10, s=5, S N R=26 d B$.

the moving sensor, we integrated the phase differences of all measurements along with the sensor motion path to build the map of sources' locations, Which results in a better estimation accuracy compared to the sensor that employed directional antennas.

\section{CONCLUSION}

Instead of using a sensor network, to overcome scalibilty issues of a sensor network, we proposed a technique that utilize two antennas mounted on a moving sensor to localize co-channel PUs. We proved that with the help of the phase difference signal between the two antennas over a period of time, we can construct a map of PUs placed in the region. We demonstrated that the distance between two antennas divided by the center frequency wavelength is the determining parameter in the proposed mapping resolution. A framework was suggested to optimize the sensor path so that the number of detectable sources being maximized. Simulation results proved that the proposed technique requires very few measurements to reconstruct source's locations map and revealed the effect of sensor path on the estimation accuracy. The results demonstrate that the method outperform a sensor network that employs array antennas, since the method can synthetically build an aperture far larger than a conventional antenna array.

\section{REFERENCES}

[1] R. G. Wiley, ELINT: The interception and analysis of radar signals. Artech House, 2006.

[2] J. Matuszewski, "The analysis of modern radar signals parameters in electronic intelligence system," in 2016 13th International Conference on Modern Problems of Radio Engineering, Telecommunications and Computer Science (TCSET). IEEE, 2016, pp. 298-302.

[3] I. Alkhouri, M. Joneidi, F. Hejazi, and N. Rahnavard, "Large-scale spectrum occupancy learning via tensor decomposition and lstm networks," in 2020 IEEE International Radar Conference (RADAR). IEEE, 2020, pp. 677-682.

[4] F. Hejazi, K. Vuckovic, and N. Rahnavard, "Dyloc: Dynamic localization for massive mimo using predictive recurrent neural networks," arXiv preprint arXiv:2101.07848, 2021.

[5] J. A. Bazerque and G. B. Giannakis, "Distributed spectrum sensing for cognitive radio networks by exploiting sparsity," IEEE Transactions on Signal Processing, vol. 58, no. 3, pp. 1847-1862, 2009.

[6] M. Joneidi, A. Zaeemzadeh, and N. Rahnavard, "Dynamic sensor selection for reliable spectrum sensing via e-optimal criterion," in 2017 IEEE 14th International Conference on Mobile Ad Hoc and Sensor Systems (MASS). IEEE, 2017, pp. 452-460.

[7] X. Fu, N. D. Sidiropoulos, and W.-K. Ma, "Tensor-based power spectra separation and emitter localization for cognitive radio," in 2014 IEEE 8th Sensor Array and Multichannel Signal Processing Workshop (SAM). IEEE, 2014, pp. 421-424.

[8] M. Joneidi, H. Yazdani, A. Vosoughi, and N. Rahnavard, "Source localization and tracking for dynamic radio cartography using directional antennas," IEEE International Conference on Sensing, Communication and Networking (SECON Workshops), 2019.

[9] G. Chalasani and S. Bhaumik, "Bearing only tracking using gausshermite filter," in 2012 7th IEEE Conference on Industrial Electronics and Applications (ICIEA). IEEE, 2012, pp. 1549-1554.

[10] F. Hejazi, M. Khalili, Y. Norouzi, and M. Nayebi, "A new pseudolinear solution to bearing-only tracking," in 2013 IEEE Radar Conference (RadarCon13). IEEE, 2013, pp. 1-4.

[11] F. Hejazi, M. Joneidi, and N. Rahnavard, "A tensor-based localization framework exploiting phase interferometry measurements," in 2020 IEEE International Radar Conference (RADAR). IEEE, 2020, pp. 554559.

[12] F. Hejazi, Y. Norouzi, and M. M. Nayebi, "Lower bound of error in aoa based passive source localization using single moving platform," in East-West Design \& Test Symposium (EWDTS 2013). IEEE, 2013, pp. $1-4$.

[13] A. Farina, "Target tracking with bearings-only measurements," Signal processing, vol. 78, no. 1, pp. 61-78, 1999.

[14] F. Hejazi, Y. Norouzi, and M. Nayebi, "Sar processing to localize lpi radars," in 2014 International Radar Conference. IEEE, 2014, pp. 1-4.

[15] T. A. Ngo, M. Tummala, and J. C. McEachen, "Wireless signal localization and collection from an airborne symmetric line array network," Oct. 23 2018, uS Patent App. 10/107,891.

[16] R. Amiri, F. Behnia, and A. Noroozi, "An efficient estimator for tdoabased source localization with minimum number of sensors," IEEE Communications Letters, vol. 22, no. 12, pp. 2499-2502, 2018.

[17] Y. Wang and Y. Wu, "An efficient semidefinite relaxation algorithm for moving source localization using tdoa and fdoa measurements," IEEE Communications Letters, vol. 21, no. 1, pp. 80-83, 2016.

[18] H. Claussen, "Methods and systems for doppler recognition aided method (dream) for source localization and separation," May 312016 , uS Patent 9,357,293.

[19] R. Amiri, H. Zamani, F. Behnia, and F. Marvasti, "Sparsity-aware target localization using tdoa/aoa measurements in distributed mimo radars," Ict Express, vol. 2, no. 1, pp. 23-27, 2016.

[20] Y. Wang and K. Ho, "Unified near-field and far-field localization for aoa and hybrid aoa-tdoa positionings," IEEE Transactions on Wireless Communications, vol. 17, no. 2, pp. 1242-1254, 2017. 
[21] Y. Y. Ang, N. Nguyen, J. P. Lie, and W. S. Gan, "Localization of harmonic source using a single moving sensor of known trajectory," in 2017 Asia-Pacific Signal and Information Processing Association Annual Summit and Conference (APSIPA ASC). IEEE, 2017, pp. 324-328.

[22] J. Ramirez Jr and J. S. Rogers, "Exploiting platform motion for passive source localization with a co-prime sampled large aperture array," The Journal of the Acoustical Society of America, vol. 143, no. 6, pp. 39463958, 2018.

[23] F. Hejazi, Y. Norouzi, and M. M. Nayebi, "Sar processing to localize lpi radars," in 2014 International Radar Conference, Oct 2014, pp. 1-4.

[24] F. Hejazikookamari, M. Mahdi Nayebi, Y. Norouzi, and E. S. Kashani, "A novel method to detect and localize lpi radars," IEEE Transactions on Aerospace and Electronic Systems, pp. 1-1, 2018.

[25] F. H. Kookamari, Y. Norouzi, and M. M. Nayebi, "Using a moving aerial platform to detect and localise a low probability of intercept radar," IET Radar, Sonar \& Navigation, vol. 11, no. 7, pp. 1062-1069, 2017.

[26] D. E. Meyer, E. Lo, S. Afshari, A. Vaughan, D. Rissolo, and F. Kuester, "Utility of low-cost drones to generate $3 \mathrm{~d}$ models of archaeological sites from multisensor data," SAA Archaeol Record Mag Soc Am Archaeol, vol. 16 , no. 2, pp. 22-24, 2016.

[27] L. Tang and G. Shao, "Drone remote sensing for forestry research and practices," Journal of Forestry Research, vol. 26, no. 4, pp. 791-797, 2015.

[28] S. Qaisar, R. M. Bilal, W. Iqbal, M. Naureen, and S. Lee, "Compressive sensing: From theory to applications, a survey," Journal of Communications and networks, vol. 15, no. 5, pp. 443-456, 2013.

[29] P. R. Gill, A. Wang, and A. Molnar, "The in-crowd algorithm for fast basis pursuit denoising," IEEE Transactions on Signal Processing, vol. 59, no. 10, pp. 4595-4605, 2011.

[30] M. A. Figueiredo, R. D. Nowak, and S. J. Wright, "Gradient projection for sparse reconstruction: Application to compressed sensing and other inverse problems," IEEE Journal of selected topics in signal processing, vol. 1, no. 4, pp. 586-597, 2007.

[31] M. A. Davenport, J. N. Laska, P. T. Boufounos, and R. G. Baraniuk, "A simple proof that random matrices are democratic," arXiv preprint arXiv:0911.0736, 2009.

[32] H. Rauhut, "Compressive sensing and structured random matrices," Theoretical foundations and numerical methods for sparse recovery, vol. 9, pp. 1-92, 2010.

[33] D. L. Donoho and M. Elad, "Optimally sparse representation in general (nonorthogonal) dictionaries via 1 minimization," Proceedings of the National Academy of Sciences, vol. 100, no. 5, pp. 2197-2202, 2003.

[34] R. Gribonval and M. Nielsen, "Sparse representations in unions of bases," 2002.

[35] A. R. Thompson, J. M. Moran, G. W. Swenson et al., Interferometry and synthesis in radio astronomy. Wiley New York et al., 1986.

[36] R. Bamler and P. Hartl, "Synthetic aperture radar interferometry," Inverse problems, vol. 14, no. 4, p. R1, 1998. 